\title{
Assessment of Knowledge and Practices of Voluntary Blood Donation Among Pharmacy Students; A Cross-Sectional Survey from Lahore, Pakistan
}

\author{
Rida Asghar, Nageen Zeeshan, Ahmed Umer Sohaib, Abu Bakar Munir, Muhammad Arslan Amjad, \\ Sharjeel Ahmed, Jamshaid Akbar* \\ Department of Pharmaceutical Sciences, The Superior College, Lahore, Pakistan
}

\author{
Authors' Contributions \\ 1,2 Conception \& Study Design, Data \\ Collection. \\ 3 Drafting, Critical Review. \\ 4 Conception \& Study Design, Critical Review. \\ 5 Data Collection. \\ 6 Data Collection, Data Analysis, Drafting. \\ 7 Data Analysis, Drafting, Critical Review.
}

\section{Article info.}

Received: December 6, 2019

Accepted: January 18, 2020

Funding Source: Nil

Conflict of Interest: Nil

Cite this article: Asghar R, Zeeshan N,

Sohaib AU, Munir AB, Amjad MA, Ahmed S,

Akbar J. Assessment of Knowledge and

Practices of Voluntary Blood Donation Among

Pharmacy Students; A Cross-Sectional Survey

from Lahore, Pakistan. RADS J Pharm Pharm

Sci. 2019; 7(4): 206-214.

*Address of Correspondence Author:

dr.jamshaidsheikh@gmail.com

\section{A B S TR AC T}

Objective: To determine the current knowledge and practices regarding voluntary blood donation among pharmacy (Pharm-D) students of Lahore, Pakistan.

Methods: A cross-sectional quantitative survey was performed among Pharm-D students of two private and two public sector universities of Lahore, Pakistan in January 2018. Total 600 students were included in the study by convenient sampling technique. Data was gathered by a self-administered well-designed questionnaire and was analyzed by using SPSS version 23.

Results: The level of knowledge among majority respondents (61.3\%) about different aspects of blood donation was found to be good. The results revealed that despite having good knowledge, a significant number of the respondents $(65.2 \%)$ were not practicing blood donation. The major reasons identified for poor blood donation practice were inconvenient environment and lack of facilities for the donors.

Conclusion: There exists a gap in converting the good knowledge of respondents into practice of voluntary blood donation. Effective programs should be initiated to promote and aware people regarding the necessity of blood donation.

Keywords: Voluntary blood donation, donors, knowledge, practice.

\section{INTRODUCTION}

Pakistan is first in terms of highest number of road accidents in Asia while ranked 48th in the world, stated by different reports [1]. Blood transfusion is the first step in the resuscitation of victims of road accidents and injuries [2]. On the other hand, blood transfusion is regularly used in cases of surgery, trauma, gastrointestinal bleeding and childbirth to replace major blood losses. Moreover, patients with genetic disorder, such as thalassemia need regular supplies of safe blood to replace their deficient blood [1].
According to the statistics, one in seven hospitalized patients need blood [3]. While many hospitals and clinics remains in constant need of blood donors. On average, our body has around 4-5 litres of blood and a person can donate blood after every 3-4 months [4]. Every year on June 14, people around the world donate blood in honor of World Blood Donor Day, a celebration to raise awareness for the need of blood and to thank all voluntary donors [5]. As stated by the World Health Organization, blood donations can help patients with life-threatening conditions to live longer, support surgeries, and help patients in emergency conditions [6]. Millions of lives can be 
saved by providing safe and adequate amount of blood in the hour of need. But the safe and good quality blood transfusion services are still under concern in developing countries that emphasizes on the need of a joint effort by Blood bank centers and medical professionals to promote safe practice of blood donation [5].

The total requirement of blood transfusion is approximately 1.5 million bags per annum in Pakistan but only $60 \%$ of this is collected in the blood collection centers [7]. The major reasons highlighted for insufficient blood donations are lack of proper knowledge and misconceptions about blood donation process [8]. Other barriers that influence the behavior of people towards blood donation are the cultural beliefs in some ethnic groups [9]. Apart from cultural myths, inconvenient environment of blood collection centers, low quality of services and fear of needle also contribute to factors related to insufficient blood donation [10]. The surveys regarding knowledge and practices may help blood donation centers to develop future policies to motivate people to donate blood regularly and to inspire non donors to start donating blood. Voluntary unpaid blood donors are safest group of blood donors and they could be a source of sustainable national blood supplies sufficient for the country's blood demand [11]. Limited studies are present that evaluated awareness and practices of voluntary blood donation among medical students that needs to expand as this population can be a very good source of quality blood if motivated to donate blood voluntarily [12].

Therefore, the present study aimed for determining the current level of knowledge and practices of Pharm-D students of Lahore regarding blood donation. By obtaining valid insight into the knowledge and practice of voluntary blood donation in the targeted area we can develop an understanding of overall practice among pharmacists.

\section{MATERIALS AND METHODS}

A cross-sectional quantitative survey was performed to evaluate the knowledge and practices of voluntary blood donation among Pharm-D (Doctor of Pharmacy) students studying at four different universities of Lahore, Pakistan. Among those four universities, two universities were public sector (Punjab University and University of Veterinary and Animal Sciences) and other two were of private sector
(University of Central Punjab and Superior University). The study was conducted for period of one month i.e. January 2018 by employing convenient random sampling technique. Total 600 Pharm-D students were enrolled in the study, 150 students from each university irrespective of gender and year of study.

A well-structured questionnaire was prepared that was divided into three sections. Section-1 was about the demographic data whereas Section-2 and Section-3 contain 15 and 9 questions regarding knowledge and practice of voluntary blood donation respectively. Data was analyzed by using SPSS version 23. Descriptive and inferential statistics were applied to tabulate the results. Chi-square test was used to investigate association between dependent and independent variables. Scoring was done for evaluating knowledge and practice level that was categorized as good and poor. Each correct response was given score 1 while every incorrect response was scored 0 . Total knowledge score ranged between 0-11 in which score from 0-6 was considered as poor knowledge while knowledge scores more than 6 was considered as good. By the same way practice was assessed where total score ranged from $0-5$. Practice scores from 0-3 was considered as poor while the score more than 3 was considered as good practice level [12].

\section{RESULTS}

Table 1 showed the demographic characteristic of the study participants. Total 600 Pharm-D students were included in the study. The mean age of the respondents was $20.1 \pm 1.46$. Most of the participants $311(51.8 \%)$ were male. Only $38(6.2 \%)$ respondents were married and most of the respondents 167 $(27.8 \%)$ were from Pharm-D $4^{\text {th }}$ professional year. Blood group $\mathrm{B}^{+}$was found most prevalent.

\section{Knowledge About Voluntary Blood Donation}

Majority of the respondents (69\%) were of the belief that regular blood donation is beneficial for health. About $43.3 \%$ of the respondents thought they will get infected if they donate blood while a significant proportion $(73.8 \%)$ of participants were of the view that screening of blood prior to blood donation is necessary. Only $41.3 \%$ of the total respondents knew about the criteria of blood donation and most of the students $(61.5 \%)$ responded that safe blood 
donations practices are available in Pakistan. Only $22.6 \%$ respondents believe that donating blood is not necessary. On the other hand, $91.2 \%$ respondents believe that donating blood saves many lives and about $54 \%$ of the respondents accepts blood donation as religious obligation (Table $\mathbf{2}$ ).

Table 1. Demographic Data of Students.

\begin{tabular}{|c|c|c|c|}
\hline \multicolumn{2}{|c|}{ Demographics } & Frequency (n) & Percentage (\%) \\
\hline \multicolumn{2}{|c|}{ Age (years) mean (SD) } & \multicolumn{2}{|c|}{$20.1( \pm 1.4)$} \\
\hline \multirow{6}{*}{ Age } & 18 & 52 & 8.7 \\
\hline & 19 & 193 & 32.2 \\
\hline & 20 & 135 & 22.5 \\
\hline & 21 & 131 & 21.8 \\
\hline & 22 & 3 & 0.5 \\
\hline & 23 & 86 & 14.3 \\
\hline \multirow{2}{*}{ Gender } & Male & 311 & 51.8 \\
\hline & Female & 289 & 48.2 \\
\hline \multirow{2}{*}{ Marital Status } & Single & 562 & 93.7 \\
\hline & Married & 38 & 6.3 \\
\hline \multirow{4}{*}{ Family Income } & $<14000$ & 46 & 7.7 \\
\hline & $<20000$ & 116 & 19.3 \\
\hline & $<30000$ & 150 & 25.0 \\
\hline & $<35000$ & 288 & 48 \\
\hline \multirow{5}{*}{ Education Status } & Pharm-D $1^{\text {st }}$ year & 144 & 24 \\
\hline & Pharm-D $2^{\text {nd }}$ year & 118 & 19.7 \\
\hline & Pharm-D $3^{\text {rd }}$ year & 88 & 14.7 \\
\hline & Pharm-D $4^{\text {th }}$ year & 167 & 27.8 \\
\hline & Pharm-D $5^{\text {th }}$ year & 83 & 13.8 \\
\hline \multirow{8}{*}{ Blood Group } & $\mathrm{A}+$ & 119 & 19.8 \\
\hline & A- & 62 & 10.3 \\
\hline & $\mathrm{B}+$ & 164 & 27.3 \\
\hline & $\mathrm{B}-$ & 54 & 9.0 \\
\hline & $\mathrm{AB}+$ & 65 & 10.8 \\
\hline & AB- & 15 & 2.5 \\
\hline & $\mathrm{O}+$ & 99 & 16.5 \\
\hline & O- & 22 & 3.7 \\
\hline
\end{tabular}

Table 2. Descriptive Analysis of Student Knowledge.

\begin{tabular}{|c|c|c|c|}
\hline Statement & $\begin{array}{c}\text { Yes } \\
\text { n (\%age) }\end{array}$ & $\begin{array}{c}\text { No } \\
\text { n (\%age) }\end{array}$ & $\begin{array}{c}\text { Don't Know } \\
\text { n (\%age) }\end{array}$ \\
\hline Regular blood donation has medical benefits? & $414(69.0)$ & $95(15.8)$ & $91(15.2)$ \\
\hline Can a person acquire infection after blood donation? & $260(43.3)$ & $237(39.5)$ & $103(17.2)$ \\
\hline Screening of the blood is necessary prior to blood donation? & $443(73.8)$ & $71(11.8)$ & $86(14.3)$ \\
\hline Do you know about blood donation criteria? & $248(41.3)$ & $236(39.3)$ & $116(19.3)$ \\
\hline Are safe blood donation measures practiced in Pakistan? & $369(61.5)$ & $89(14.8)$ & $142(23.7)$ \\
\hline Is donating blood unnecessary? & $136(22.6)$ & $410(68.3)$ & $54(9.0)$ \\
\hline Donating blood saves many precious lives? & $547(91.2)$ & $36(6.0)$ & $17(2.8)$ \\
\hline $\begin{array}{l}\text { "More blood more life" this theme is right for world blood donor } \\
\text { day? }\end{array}$ & $473(78.8)$ & $66(11.0)$ & $61(10.2)$ \\
\hline Are males more likely to donate blood than female? & $467(77.8)$ & $52(8.7)$ & $81(13.5)$ \\
\hline Religion urges us to donate blood? & $324(54.0)$ & $118(19.7)$ & $158(26.3)$ \\
\hline Pregnant or lactating mothers cannot donate blood? & $315(52.5)$ & $146(24.3)$ & $139(23.2)$ \\
\hline
\end{tabular}


Table 3. Association Between Knowledge and Demographics.

\begin{tabular}{|c|c|c|c|c|c|c|c|c|}
\hline \multirow[t]{2}{*}{ Statement } & \multirow[t]{2}{*}{ Response } & \multicolumn{5}{|c|}{ Gender } & Total & P-Value \\
\hline & & \multicolumn{2}{|c|}{ Male } & \multicolumn{3}{|c|}{ Female } & & \\
\hline \multirow{4}{*}{$\begin{array}{c}\text { Regular blood } \\
\text { donation has } \\
\text { medical benefits? }\end{array}$} & Yes & \multicolumn{2}{|c|}{$232(74.6 \%)$} & \multicolumn{3}{|c|}{$182(63.0 \%)$} & $\begin{array}{c}414 \\
(69.0 \%)\end{array}$ & \multirow{4}{*}{0.002} \\
\hline & No & \multicolumn{2}{|c|}{$34(10.9 \%)$} & \multicolumn{3}{|c|}{$61(21.1 \%)$} & $\begin{array}{c}95 \\
(15.8 \%)\end{array}$ & \\
\hline & Don’t Know & \multicolumn{2}{|c|}{$45(14.5 \%)$} & \multicolumn{3}{|c|}{$46(15.9 \%)$} & $\begin{array}{c}91 \\
(15.2 \%)\end{array}$ & \\
\hline & Total & \multicolumn{2}{|c|}{$311(100 \%)$} & \multicolumn{3}{|c|}{$289(100 \%)$} & $\begin{array}{c}600 \\
(100 \%)\end{array}$ & \\
\hline \multirow{4}{*}{$\begin{array}{l}\text { Can a person } \\
\text { acquire infection } \\
\text { after blood } \\
\text { donation? }\end{array}$} & Yes & \multicolumn{2}{|c|}{$113(36.3 \%)$} & \multicolumn{3}{|c|}{$147(50.9 \%)$} & $\begin{array}{c}260 \\
(43.3 \%)\end{array}$ & \multirow{4}{*}{$<0.001$} \\
\hline & No & \multicolumn{2}{|c|}{$145(46.6 \%)$} & \multicolumn{3}{|c|}{$92(31.8 \%)$} & $\begin{array}{c}237 \\
(39.5 \%)\end{array}$ & \\
\hline & Don't Know & \multicolumn{2}{|c|}{$53(17.0 \%)$} & \multicolumn{3}{|c|}{$50(17.3 \%)$} & $\begin{array}{c}103 \\
(17.2 \%)\end{array}$ & \\
\hline & Total & \multicolumn{2}{|c|}{$311(100 \%)$} & \multicolumn{3}{|c|}{$289(100 \%)$} & $\begin{array}{c}600 \\
(100 \%)\end{array}$ & \\
\hline \multirow{4}{*}{$\begin{array}{c}\text { Are males more } \\
\text { likely to donate } \\
\text { blood than females? }\end{array}$} & Yes & \multicolumn{2}{|c|}{$257(82.6 \%)$} & \multicolumn{3}{|c|}{$210(72.7 \%)$} & $\begin{array}{c}467 \\
(77.8 \%)\end{array}$ & \\
\hline & No & \multicolumn{2}{|c|}{$15(4.8 \%)$} & \multicolumn{3}{|c|}{$37(12.8 \%)$} & $\begin{array}{c}52 \\
(8.7 \%)\end{array}$ & م001 \\
\hline & Don't Know & \multicolumn{2}{|c|}{$39(12.5 \%)$} & & $42(14.5 \%)$ & & $\begin{array}{c}81 \\
(13.5 \%)\end{array}$ & 0.007 \\
\hline & Total & 311( & $00 \%)$ & & $289(100 \%)$ & & $\begin{array}{c}600 \\
(100 \%)\end{array}$ & \\
\hline & Yes & $196(6$ & $3.0 \%)$ & & $128(44.3 \%)$ & & $\begin{array}{c}324 \\
(54.0 \%)\end{array}$ & \\
\hline Religion urges us to & No & $49(1$ & $.8 \%)$ & & $69(23.9 \%)$ & & $\begin{array}{c}118 \\
(19.7 \%)\end{array}$ & \\
\hline donate blood? & Don't Know & $66(2$ & $.2 \%)$ & & $92(31.8 \%)$ & & $\begin{array}{c}158 \\
(26.3 \%)\end{array}$ & $<0.001$ \\
\hline & Total & 311( & $00 \%)$ & & $289(100 \%)$ & & $\begin{array}{c}600 \\
(100 \%)\end{array}$ & \\
\hline & & & & Jcation Ste & & & & \\
\hline & & $\begin{array}{l}\text { Pharm-D } \\
1^{\text {st }} \text { year }\end{array}$ & $\begin{array}{l}\text { Pharm-D } \\
2^{\text {nd }} \text { year }\end{array}$ & $\begin{array}{l}\text { Pharm-D } \\
3^{\text {rd }} \text { year }\end{array}$ & $\begin{array}{l}\text { Pharm-D } \\
4^{\text {th }} \text { year }\end{array}$ & $\begin{array}{l}\text { Pharm-D } \\
5^{\text {th }} \text { year }\end{array}$ & & \\
\hline Screening of your & Yes & $\begin{array}{c}116 \\
(80.6 \%)\end{array}$ & $\begin{array}{c}81 \\
(68.66 \%)\end{array}$ & $\begin{array}{c}55 \\
(62.5 \%)\end{array}$ & $\begin{array}{c}123 \\
(73.7 \%)\end{array}$ & $\begin{array}{c}68 \\
(81.9 \%)\end{array}$ & $\begin{array}{c}443 \\
(73.8 \%)\end{array}$ & \\
\hline $\begin{array}{l}\text { blood prior to blood } \\
\text { donation is }\end{array}$ & No & $9(6.2 \%)$ & $\begin{array}{c}21 \\
(17.8 \%)\end{array}$ & $\begin{array}{c}18 \\
(20.5 \%)\end{array}$ & $14(8.4 \%)$ & $9(10.8 \%)$ & $\begin{array}{c}71 \\
(11.8 \%)\end{array}$ & 0.003 \\
\hline necessary & Don't Know & $\begin{array}{c}19 \\
(13.2 \%)\end{array}$ & $\begin{array}{c}16 \\
(13.6 \%)\end{array}$ & $15(17 \%)$ & $30(18 \%)$ & $6(7.2 \%)$ & $\begin{array}{c}86 \\
(14.3 \%)\end{array}$ & \\
\hline & Total & $\begin{array}{c}144 \\
(100 \%)\end{array}$ & $\begin{array}{c}118 \\
(100 \%)\end{array}$ & $\begin{array}{c}88 \\
(100 \%)\end{array}$ & $\begin{array}{c}167 \\
(100 \%)\end{array}$ & $\begin{array}{c}83 \\
(100 \%)\end{array}$ & $\begin{array}{c}600 \\
(100 \%)\end{array}$ & \\
\hline Are safe blood & Yes & $\begin{array}{c}81 \\
(56.2 \%)\end{array}$ & $\begin{array}{c}85 \\
(72.0 \%)\end{array}$ & $\begin{array}{c}55 \\
(62.5 \%)\end{array}$ & $\begin{array}{c}89 \\
(53.3 \%)\end{array}$ & $\begin{array}{c}59 \\
(71.1 \%)\end{array}$ & $\begin{array}{c}369 \\
(61.5 \%)\end{array}$ & \\
\hline $\begin{array}{l}\text { practiced in } \\
\text { Pakistan? }\end{array}$ & No & $\begin{array}{c}28 \\
(19.4 \%)\end{array}$ & $13(11 \%)$ & $\begin{array}{c}14 \\
(15.9 \%)\end{array}$ & $\begin{array}{c}24 \\
(14.4 \%)\end{array}$ & $10(12 \%)$ & $\begin{array}{c}89 \\
(14.8 \%)\end{array}$ & 0.015 \\
\hline
\end{tabular}




\begin{tabular}{|c|c|c|c|c|c|c|c|c|}
\hline & Don't Know & $\begin{array}{c}35 \\
(24.3 \%)\end{array}$ & $\begin{array}{c}20 \\
(16.9 \%)\end{array}$ & $\begin{array}{c}19 \\
(21.6 \%)\end{array}$ & $\begin{array}{c}54 \\
(32.3 \%)\end{array}$ & $\begin{array}{c}14 \\
(16.9 \%)\end{array}$ & $\begin{array}{c}142 \\
(23.7 \%)\end{array}$ & \\
\hline & Total & $\begin{array}{c}144 \\
(100 \%)\end{array}$ & $\begin{array}{c}118 \\
(100 \%)\end{array}$ & $\begin{array}{c}88 \\
(100 \%)\end{array}$ & $\begin{array}{c}167 \\
(100 \%)\end{array}$ & $\begin{array}{c}83 \\
(100 \%)\end{array}$ & $\begin{array}{c}600 \\
(100 \%)\end{array}$ & \\
\hline \multirow{4}{*}{$\begin{array}{l}\text { Is donating blood } \\
\text { unnecessary? }\end{array}$} & Yes & $\begin{array}{c}20 \\
(13.9 \%)\end{array}$ & $\begin{array}{c}36 \\
(30.5 \%)\end{array}$ & $\begin{array}{c}35 \\
(39.8 \%)\end{array}$ & 35 (21\%) & $10(12 \%)$ & $\begin{array}{c}136 \\
(22.7 \%)\end{array}$ & \multirow{4}{*}{$<0.001$} \\
\hline & No & $\begin{array}{c}111 \\
(77.1 \%)\end{array}$ & $\begin{array}{c}78 \\
(66.1 \%)\end{array}$ & $\begin{array}{c}39 \\
(44.3 \%)\end{array}$ & $\begin{array}{c}113 \\
(67.7 \%)\end{array}$ & $\begin{array}{c}69 \\
(83.1 \%)\end{array}$ & $\begin{array}{c}410 \\
(68.3 \%)\end{array}$ & \\
\hline & Don't Know & $13(9 \%)$ & $4(3.4 \%)$ & $\begin{array}{c}14 \\
(15.9 \%)\end{array}$ & $\begin{array}{c}19 \\
(11.4 \%)\end{array}$ & $4(4.8 \%)$ & $54(9 \%)$ & \\
\hline & Total & $\begin{array}{c}144 \\
(100 \%) \\
\end{array}$ & $\begin{array}{c}118 \\
(100 \%) \\
\end{array}$ & $\begin{array}{c}88 \\
(100 \%) \\
\end{array}$ & $\begin{array}{c}167 \\
(100 \%) \\
\end{array}$ & $\begin{array}{c}83 \\
(100 \%) \\
\end{array}$ & $\begin{array}{c}600 \\
(100 \%)\end{array}$ & \\
\hline \multirow{4}{*}{$\begin{array}{l}\text { Donating blood } \\
\text { saves many } \\
\text { precious lives }\end{array}$} & Yes & $\begin{array}{c}138 \\
(95.8 \%)\end{array}$ & $\begin{array}{c}103 \\
(87.3 \%)\end{array}$ & $\begin{array}{c}71 \\
(80.7 \%)\end{array}$ & $\begin{array}{c}153 \\
(91.6 \%)\end{array}$ & $\begin{array}{c}82 \\
(98.8 \%)\end{array}$ & $\begin{array}{c}547 \\
(91.2 \%)\end{array}$ & \multirow{4}{*}{$<0.001$} \\
\hline & No & $6(4.2 \%)$ & $\begin{array}{c}12 \\
(10.2 \%)\end{array}$ & $\begin{array}{c}12 \\
(13.6 \%)\end{array}$ & $6(3.6 \%)$ & $0(0 \%)$ & $36(6 \%)$ & \\
\hline & Don't Know & $0(0 \%)$ & 3 (2.5\%) & 5 (5.7\%) & 8 (4.8\%) & $1(1.2 \%)$ & $\begin{array}{c}17 \\
(2.8 \%)\end{array}$ & \\
\hline & Total & $\begin{array}{c}144 \\
(100 \%)\end{array}$ & $\begin{array}{c}118 \\
(100 \%)\end{array}$ & $\begin{array}{c}88 \\
(100 \%)\end{array}$ & $\begin{array}{c}167 \\
(100 \%)\end{array}$ & $\begin{array}{c}83 \\
(100 \%)\end{array}$ & $\begin{array}{c}600 \\
(100 \%)\end{array}$ & \\
\hline \multirow{4}{*}{$\begin{array}{l}\text { "More blood More } \\
\text { life" is this theme } \\
\text { right for World Blood } \\
\text { Donor Day? }\end{array}$} & Yes & $\begin{array}{c}114 \\
(79.2 \%)\end{array}$ & $\begin{array}{c}99 \\
(83.9 \%)\end{array}$ & $\begin{array}{c}60 \\
(68.2 \%)\end{array}$ & $\begin{array}{c}127 \\
(76 \%)\end{array}$ & 73 (88\%) & $\begin{array}{c}473 \\
(78.8 \%)\end{array}$ & \multirow{4}{*}{0.009} \\
\hline & No & $13(9 \%)$ & $\begin{array}{c}14 \\
(11.9 \%)\end{array}$ & $\begin{array}{c}17 \\
(19.3 \%)\end{array}$ & $16(9.6 \%)$ & $6(7.2 \%)$ & $\begin{array}{c}66 \\
(11 \%)\end{array}$ & \\
\hline & Don't Know & $\begin{array}{c}17 \\
(11.8 \%) \\
\end{array}$ & 5 (4.2\%) & $\begin{array}{c}11 \\
(12.5 \%) \\
\end{array}$ & $\begin{array}{c}24 \\
(14.44 \%) \\
\end{array}$ & $4(4.8 \%)$ & $\begin{array}{c}61 \\
(10.2 \%) \\
\end{array}$ & \\
\hline & Total & $\begin{array}{c}144 \\
(100 \%)\end{array}$ & $\begin{array}{c}118 \\
(100 \%)\end{array}$ & $\begin{array}{c}88 \\
(100 \%)\end{array}$ & $\begin{array}{c}167 \\
(100 \%)\end{array}$ & $\begin{array}{c}83 \\
(100 \%)\end{array}$ & $\begin{array}{c}600 \\
(100 \%)\end{array}$ & \\
\hline \multirow{4}{*}{$\begin{array}{c}\text { Are males more } \\
\text { likely to donate } \\
\text { blood than females? }\end{array}$} & Yes & $\begin{array}{c}110 \\
(76.4 \%)\end{array}$ & $\begin{array}{c}99 \\
(83.9 \%)\end{array}$ & $\begin{array}{c}56 \\
(63.6 \%)\end{array}$ & $\begin{array}{c}125 \\
(74.9 \%)\end{array}$ & $\begin{array}{c}77 \\
(92.8 \%)\end{array}$ & $\begin{array}{c}467 \\
(77.8 \%)\end{array}$ & \multirow{4}{*}{$<0.001$} \\
\hline & No & 7 (4.9\%) & 7 (5.9\%) & $\begin{array}{c}17 \\
(19.3 \%)\end{array}$ & 20 (12\%) & $1(1.2 \%)$ & $\begin{array}{c}52 \\
(8.7 \%)\end{array}$ & \\
\hline & Don't Know & $\begin{array}{c}27 \\
(18.8 \%)\end{array}$ & $\begin{array}{c}12 \\
(10.2 \%)\end{array}$ & $15(17 \%)$ & $\begin{array}{c}22 \\
(13.2 \%)\end{array}$ & $5(6 \%)$ & $\begin{array}{c}81 \\
(13.5 \%)\end{array}$ & \\
\hline & Total & $\begin{array}{c}144 \\
(100 \%)\end{array}$ & $\begin{array}{c}118 \\
(100 \%)\end{array}$ & $\begin{array}{c}88 \\
(100 \%) \\
\end{array}$ & $\begin{array}{c}167 \\
(100 \%) \\
\end{array}$ & $\begin{array}{c}83 \\
(100 \%) \\
\end{array}$ & $\begin{array}{c}600 \\
(100 \%) \\
\end{array}$ & \\
\hline \multirow{4}{*}{$\begin{array}{l}\text { Religion urges us to } \\
\text { donate blood? }\end{array}$} & Yes & $\begin{array}{c}79 \\
(54.9 \%)\end{array}$ & $\begin{array}{c}62 \\
(52.5 \%)\end{array}$ & $\begin{array}{c}50 \\
(56.8 \%)\end{array}$ & $\begin{array}{c}83 \\
(49.7 \%)\end{array}$ & $\begin{array}{c}50 \\
(60.2 \%)\end{array}$ & $\begin{array}{c}324 \\
(54 \%)\end{array}$ & \multirow{4}{*}{$<0.001$} \\
\hline & No & $\begin{array}{c}14 \\
(9.7 \%)\end{array}$ & $\begin{array}{c}38 \\
(32.2 \%)\end{array}$ & 22 (25\%) & $\begin{array}{c}29 \\
(17.4 \%)\end{array}$ & $\begin{array}{c}15 \\
(18.1 \%)\end{array}$ & $\begin{array}{c}118 \\
(19.7 \%)\end{array}$ & \\
\hline & Don't Know & $\begin{array}{c}51 \\
(35.4 \%) \\
\end{array}$ & $\begin{array}{c}18 \\
(15.3 \%)\end{array}$ & $\begin{array}{c}16 \\
(18.2 \%)\end{array}$ & $\begin{array}{c}55 \\
(32.9 \%) \\
\end{array}$ & $\begin{array}{c}18 \\
(21.7 \%) \\
\end{array}$ & $\begin{array}{c}158 \\
(26.3 \%) \\
\end{array}$ & \\
\hline & Total & $\begin{array}{c}144 \\
(100 \%)\end{array}$ & $\begin{array}{c}118 \\
(100 \%)\end{array}$ & $\begin{array}{c}88 \\
(100 \%)\end{array}$ & $\begin{array}{c}167 \\
(100 \%)\end{array}$ & $\begin{array}{c}83 \\
(100 \%)\end{array}$ & $\begin{array}{c}600 \\
(100 \%)\end{array}$ & \\
\hline
\end{tabular}

\section{Association Between Knowledge and Demographics of Participants}

Gender of the study participants was found to be statistically associated with the response regarding knowledge of voluntary blood donation as majority of the male participants (74.6\%) showed the better knowledge about the benefits of blood donation as compared to females $(p=0.002)$. About $46.6 \%$ males knew that donating blood will not infect the person $(p<0.001)$ and majority $(82.6 \%)$ of them stated that males more often donate blood $(p=0.001)$ as compare to females.

A difference in responses was also observed among participants based on their educational year of Pharm-D. Most students of final year (81.9\%) responded that blood screening is important before 
donation of blood $(p=0.003)$. About $72 \%$ of the students of Pharm-D $2^{\text {nd }}$ year responded that safe blood donation practices are available in Pakistan $(p=0.015)$ while majority of the respondents from Pharm-D final year(83.1\%) believe that donating blood is not unnecessary $(p<0.001)$ and almost all $(98.8 \%)$ of them knew that donating blood saves many lives $(p<0.001)$. The students of final year $(92.8 \%)$ responded that males more often donate blood than females $(p<0.001)$ and about $60.2 \%$ were of the view that donating blood is the religious obligation $(p<0.001)$ (Table 3$)$.

\section{Practice of Voluntary Blood Donation}

Out of the total only $27.8 \%$ had ever donated blood. Most students $(75 \%)$ responded that they will not donate blood as a paid donor. Surprisingly, about
$63.8 \%$ of them had never attended any educational or awareness program regarding blood donation and interestingly $34.3 \%$ of the students explained fear of pain while donating blood. About $(77 \%)$ were willing to donate blood in their future (Table 4).

\section{Association Between Practice and Demographics}

In comparison to the male students, blood donation practice was found poor among females where only $(16.6 \%)$ were reported who donated blood once in life $(p<0.001)$. A significant proportion of the females $(45.3 \%)$ were not donating blood due to the fear of pain $(p<0.001)$.

Comparatively most of the students of Pharm-D $1^{\text {st }}$ year $(72.2 \%)$ do not had a fear of pain while donating blood ( $p=0.019)$ and most of them $(93.1 \%)$ were willing to donate blood in future $(<0.001)$ (Table 5).

Table 4. Practice of Voluntary Blood Donation.

\begin{tabular}{|c|c|c|}
\hline Statement & $\begin{array}{c}\text { Yes } \\
\mathbf{n}(\% \mathrm{age})\end{array}$ & $\begin{array}{c}\text { No } \\
\mathbf{n}(\% \mathrm{oge})\end{array}$ \\
\hline Have you donated blood before? & $167(27.8)$ & $433(72.2)$ \\
\hline If you got paid, will you donate blood & $150(25.0)$ & $450(75.0)$ \\
\hline Have you ever attended any educational program on blood \\
donation? & $217(36.2)$ & $383(63.8)$ \\
\hline Do you afraid of pain while donating blood? & $206(34.3)$ & $394(65.7)$ \\
\hline You are willing to donate blood in future? & $462(77.0)$ & $138(23.0)$ \\
\hline
\end{tabular}

Table 5. Association Between Practice and Demographics.

\begin{tabular}{|c|c|c|c|c|c|}
\hline \multirow[t]{2}{*}{ Statement } & \multirow[t]{2}{*}{ Response } & \multicolumn{2}{|c|}{ Gender } & \multirow[t]{2}{*}{ Total } & \multirow[t]{2}{*}{ P -Value } \\
\hline & & Male & Female & & \\
\hline \multirow{3}{*}{$\begin{array}{c}\text { Have you } \\
\text { donated } \\
\text { blood before? }\end{array}$} & Yes & $119(38.3 \%)$ & $48(16.6 \%)$ & $\begin{array}{c}167 \\
(27.8 \%)\end{array}$ & \multirow{3}{*}{$<0.001$} \\
\hline & No & $192(61.7 \%)$ & $241(83.4 \%)$ & $\begin{array}{c}433 \\
(72.2 \%)\end{array}$ & \\
\hline & Total & $311(100.0 \%)$ & $289(100.0 \%)$ & $\begin{array}{c}600 \\
(100.0 \%)\end{array}$ & \\
\hline \multirow{3}{*}{$\begin{array}{l}\text { Do you afraid } \\
\text { of pain while } \\
\text { donating } \\
\text { blood? }\end{array}$} & Yes & $75(24.1 \%)$ & $131(45.3 \%)$ & $\begin{array}{c}206 \\
(34.3 \%) \\
\end{array}$ & \multirow{3}{*}{$<0.001$} \\
\hline & No & $236(75.9 \%)$ & $158(54.7 \%)$ & $\begin{array}{c}394 \\
(65.7 \%)\end{array}$ & \\
\hline & Total & $311(100.0 \%)$ & $289(100.0 \%)$ & $\begin{array}{c}600 \\
(100.0 \%)\end{array}$ & \\
\hline \multirow{3}{*}{$\begin{array}{c}\text { Are you } \\
\text { willing to } \\
\text { donate blood } \\
\text { in future? }\end{array}$} & Yes & $256(82.3 \%)$ & $206(71.3 \%)$ & $\begin{array}{c}462 \\
(77.0 \%)\end{array}$ & \multirow{3}{*}{0.001} \\
\hline & No & $55(17.7 \%)$ & $83(28.7 \%)$ & $\begin{array}{c}138 \\
(23.0 \%)\end{array}$ & \\
\hline & Total & $311(100.0 \%)$ & $289(100.0 \%)$ & $\begin{array}{c}600 \\
(100.0 \%)\end{array}$ & \\
\hline
\end{tabular}




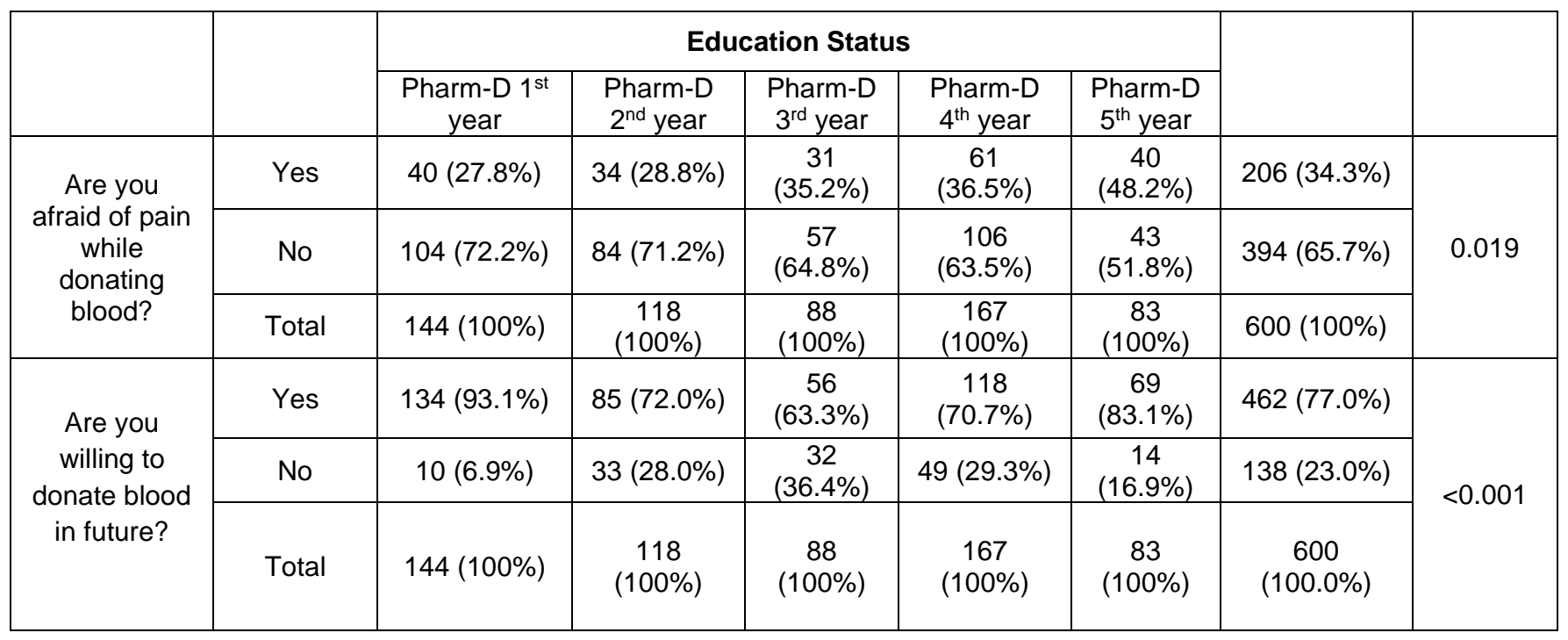

Table 6. Level of Knowledge.

\begin{tabular}{|c|c|c|}
\hline Knowledge & COV & Frequency (\%) \\
\hline Poor & $0-6$ & $232(38.7 \%)$ \\
\hline Good & $>6$ & $368(61.3 \%)$ \\
\hline Practice & COV & Frequency (\%) \\
\hline Poor & $0-3$ & $391(65.2 \%)$ \\
\hline Good & $>3$ & $209(34.8 \%)$ \\
\hline
\end{tabular}

${ }^{*} \mathrm{COV}=$ Cut of Value

\section{Level of Knowledge}

The overall knowledge of most of the respondents $(61.3 \%)$ about different aspects of blood donation found to be good but the level of practice was reported poor among (65.2\%) of the studied population (Table 6).

\section{DISCUSSION}

The present study aimed to assess the knowledge and practice of voluntary blood donation among students of Lahore. The results obtained by the study revealed that sufficient number of students has good knowledge about blood donation but most of the respondents are not practicing the blood donation activities. Different countries of the world like Saudi Arabia, Jordan, Nepal, India and Ethiopia had conducted various studies on knowledge and practice of voluntary blood donation. A study conducted in Saudi Arabia reported results contrary to the results of our study. The results suggest that there was lack of basic knowledge of blood donation criteria among population [13]. Another study conducted in King
Abdul Aziz Medical City also showed results contrary to our study. The reasons for poor level of knowledge was lack of general information regarding blood donation policies among the surveyed individuals [5]. A study conducted in Iran showed results different to our study, as their knowledge about blood donation was unsatisfactory [14]. Different studies conducted in India showed similar trend about knowledge and practice regarding blood donation [15, 16]. A study conducted in Ethiopia also revealed similar results about knowledge and practice of blood donation [17].

A study conducted among students of Nigeria showed similar results of knowledge and practice about blood donation. Although the results showed poor level of practice but among the donor participants the ratio for male and female donors was same, contrary to our study in which males were more likely to donate blood than females [18]. Similar results about knowledge and practice of blood donation was revealed by a study conducted among medical undergraduates in India [19]. Another report conducted in medical institute of neighbor country India revealed good level of knowledge and practice among the study 
participants. The reason for good level of practice was the concern of the participants for the well-being of others [20]. A study conducted in Saudi Arabia shows that the percentage of females with good level of knowledge was more while our results showed males were more aware than females [16].

Various studies conducted around the country reported similar results regarding knowledge of blood donation [11, 12, 21, 22], but their level of practice was comparatively good than the participants of our study. The reason for practicing blood donation was to help the relatives and friends in the hour of need. Most of the studies conducted in Pakistan reported that males were more likely to donate blood than females, this result corroborated with the result of our study [2, 12, 21, 23]. Different studies reported that participants responded to donate more blood in their future, this is also in accordance with the results of our study [11, 22]. A study survey from rural areas of Khyber Pakhtunkhwa revealed contrary results about blood donation knowledge among rural population. There was an association between the knowledge and gender, because male participants were more aware than females. The reason reported for poor knowledge was that no one ever talked to them about blood donation [24].

Although the participants had good knowledge, their level of practice is not up to the mark. Most of the people donate blood only when their relatives were in need of blood. So there is a requirement for inspiration among the youth by sorting out instructive workshops about blood donation. Appropriate measures ought to be adopted for actual blood screening, teaching the public about the health advantages of consistent blood donation and propelling the individuals for this obligation. A major gap is observed in practice of female donors. In order to increase the practices of blood donation it is necessary to build a safe and positive image of the donor in public so that females can equally participate in this activity. Convenience of approach to blood donation centers and provision of basic facilities are necessary to be provided to encourage female donors. Medical students and health care institutes can play pivotal role in creating awareness programs on voluntary blood donations among community, especially females. Government can also play its role in promoting blood donation practices by arranging blood donation camps with proper basic facilities in public sectors and by conducting awareness programs about blood donation among the general population of the country.

\section{CONCLUSION}

Study concluded that the level of knowledge among pharmacy students was good but there exists a gap in converting that knowledge into practice that needs to be fully addressed. Inconvenient environment and lack of screening facilities were the major reasons for poor practice of blood donation.

\section{REFERENCES}

1. Independent News Pakistan (INP). Pakistan has the highest number of road accidents in Asia. 2017; [updated February 2, 2017]. Available from: https://www.pakistantoday.com.pk/2017/02/02/paki stan-has-the-highest-number-of-road-accidents-inasia/.

2. Shera MT, Haider A, Bareeqa SB, Ahmed SI, Junaid MM, Khalid Z, et al. Factors Refraining Medical Students from Blood Donation; a Tertiary Care Experience from a Medical College of Pakistan. ARC Journal of Public Health and Community Medicine. 2017; 2(3):13-20.

3. Arrive alive. Road Safety and the Need for Blood. 2018; [cited 2018]. Available from: https://www.arrivealive.mobi/blood-products.

4. Wilson K. Blood Donation is Free and Saves Lives 2018 [cited 201821 oct]. Available from: https://www.gov.bm/articles/blood-donation-freeand-saves-lives.

5. Alfouzan N. Knowledge, attitudes, and motivations towards blood donation among King Abdulaziz Medical City Population. Int J Fam Med. 2014; 2014:539670.

6. Towards $100 \%$ Voluntary Blood Donation: A Global Framework for Action. Geneva: World Health Organization; 2010. Available from: https://www.ncbi.nlm.nih.gov/books/NBK305667/.

7. The News. Dire need of motivation for blood donation in Pakistan 2018 [cited 2018 29]. Available www.thenews.com.pk/print/329553-dire-need-ofmotivation-for-blood-donation-in-pakistan.

8. Bilal M, Haseeb A, Zahid I, Lashkerwala SS, Saeeduddin F, Saad M, et al. Knowledge, Attitudes and Perceptions Among Non-Blood Donor Female Health Care Professionals. Glob J Health Sci. 2016; 8(4):203-11.

9. Wilkinson A, Gupta RS. Perceptions of Blood Donation amongst the Youth. Panacea J Med Sci. 2016; 6(2):79-82.

10. Dubey A, Sonker A, Chaurasia R, Chaudhary R. Knowledge, attitude and beliefs of people in North India regarding blood donation. Blood Transfus. 2014; 12 Suppl 1:s21-7. 
11. Latif MZ, Riaz H, Mujtaba SWA, Nizami R. Blood Donation; Knowledge, Attitude and Practice of Medical Students. The Professional Medical Journal. 2017; 24(3):370-4.

12. Ahmed Z, Zafar M, Khan AA, Anjum MU, Siddiqui MA. Knowledge, attitude and practices about blood donation among undergraduate medical students in Karachi. J Infect Dis Ther. 2014; 2(2):134.

13. Alam M, Masalmeh DBD. Knowledge, attitudes and practices regarding blood donation among the Saudi population. Saudi Med J. 2004; 25(3):31821.

14. Safizadeh $H$, Pourdamghan N, Mohamadi B. University students awareness and attitude towards blood donation in Kerman City. Iranian J Blood Cancer. 2009; 1(3):107-10.

15. Giri PA, Phalke DB. Knowledge and Attitude about Blood Donation Amongst Undergraduate Students of Pravara Institute of Medical Sciences Deemed University of Central India. Ann Trop Med Public Health. 2012; 5(6):569-73.

16. Sabu KM, Remya A, Binu VS, Vivek $R$. Knowledge, Attitude and Practice on Blood Donation among Health Science Students in a University campus, South India. Online J Health Allied Sci. 2011;10(2):1-3.

17. Jemberu YA, Esmael A, Ahmed KY. Knowledge, attitude and practice towards blood donation and associated factors among adults in Debre Markos town, Northwest Ethiopia. BMC Hematol. 2016; 16(1):23.

18. Salaudeen AG, Odeh E. Knowledge and behavior towards voluntary blood donation among students of a tertiary institution in Nigeria. Niger $\mathrm{J}$ Clin Pract. 2011; 14(3):303-7.

19. Patil J, bin Harunarasid AA, binti Azmi N, bin Mohd Junit $\mathrm{AH}$, bin Suaimi MH, Kumar N. A study on knowledge, attitude and practice of voluntary blood donation among the medical undergraduate students. Res J Pharm Biol Chem Sci. 2016; 7(5):2408-12.

20. Das K, Sachdev S, Kaur B, Singh Cl, Nongbri D. Knowledge, Attitude and Practices of Blood Donors toward Blood Donation. J Postgrad Med Educ Res. 2014; 48(3):123.

21. Malik MR, Majid S, Laghari MS. Determinants of blood donation behaviour of general public in Pakistan. Pak J Med Health Sci. 2010; 4(1):13742.

22. Mirza H, Khan F, Naeem FJ, Ashraf B. Blood Safety and Donation Knowledge, Attitude and Practice (KAP) among 1st Year Medical Students at LMDC, Lahore. Pak J Med Health Sci. 2015; 9(3):992-4.

23. Baig M, Habib $H$, Haji AH, Alsharief FT, Noor AM, Makki RG. Knowledge, misconceptions and motivations towards blood donation among university students in KSA. Pak J Med Sci. 2013; 29(6):1295-9.

24. Sethi H, Ayaz M, Niaz P, Sahidullah G, Aziz S, Shah $\mathrm{N}$, et al. Awareness of voluntary blood donation among the general public visiting a rural Hospital of Peshawar, Khyber Pakhtunkhwa. J Med Students. 2015; 1(2):3-11. 